\title{
The presence of multiple variants affects the clinical phenotype and prognosis in left ventricular noncompaction after surgery
}

\author{
Keiichi Hirono, MD, PhD, ${ }^{\mathrm{a}}$ Takehiko Sakai, MD, ${ }^{\mathrm{b}}$ Yukiko Hata, $\mathrm{PhD},{ }^{\mathrm{c}}$ and Naonori Nishida, $\mathrm{MD}, \mathrm{PhD},{ }^{\mathrm{a}}$ \\ Toyama and Fukui, Japan
}

\author{
From the Departments of ${ }^{\mathrm{a}}$ Pediatrics, and ${ }^{\mathrm{c}}$ Legal Medicine, Graduate School of Medicine, University of Toyama, \\ Toyama; and ${ }^{\mathrm{b}}$ Department of Pediatrics, Fukui Prefectural Hospital, Fukui, Japan. \\ Disclosures: Authors have nothing to disclose with regard to commercial support. \\ Received for publication Sept 15, 2017; accepted for publication Oct 18, 2017; available ahead of print Dec 2, \\ 2017. \\ Address for reprints: Keiichi Hirono, MD, PhD, Department of Pediatrics, Graduate School of Medicine, Univer- \\ sity of Toyama, 2630 Sugitani, Toyama City, Toyama, 930-0194 Japan (E-mail: khirono1973@gmail.com). \\ J Thorac Cardiovasc Surg 2018;155:e101-9 \\ $0022-5223 / \$ 36.00$ \\ Copyright $\subset 2017$ by The American Association for Thoracic Surgery \\ https://doi.org/10.1016/j.jtcvs.2017.10.053
}

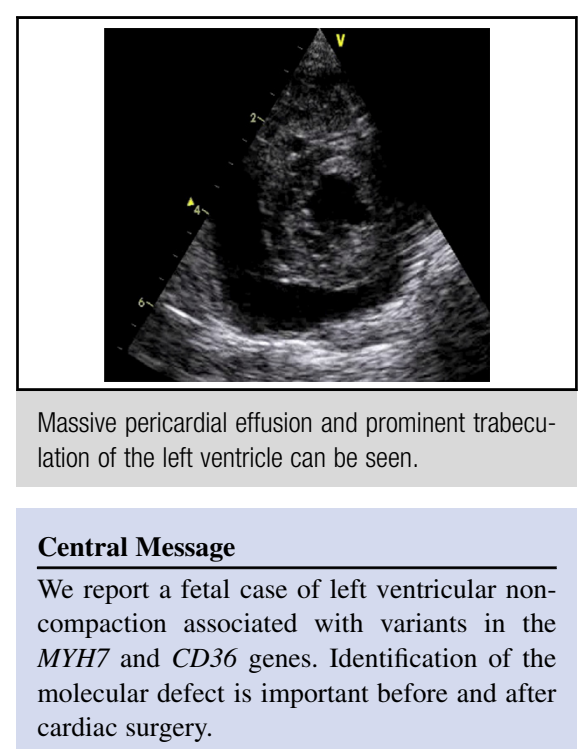

Left ventricular noncompaction (LVNC) is a hereditary cardiomyopathy morphologically characterized by numerous prominent trabeculations and deep intertrabecular recesses communicating with the left ventricular cavity. ${ }^{1}$ Here, we report the first case of prenatal echocardiography diagnosis of LVNC, which deteriorated after surgical intervention and was associated with variants in the $M Y H 7$ and $C D 36$ genes.

\section{CASE REPORT}

A 30-year-old multigravida with singleton clomiphene pregnancy was referred at 26 weeks of gestation due to the existence of ascites, and fetal echocardiography revealed that the fetus had LVNC and double outlet of the right ventricle, in addition to the interruption of the aorta and aortic stenosis (Figure 1). The patient was born as a full-term female infant with a birth weight of $2.53 \mathrm{~kg}$ and Apgar scores of 4 and 9 at 1 and 5 minutes, respectively. No other extracardiac anomalies were observed. No postnatal resuscitation was required, and intravenous prostaglandin E1 was started to retain ductus arteriosus.

As the baby became gradually symptomatic with tachypnea and tachycardia at 6 days of age, she underwent the bilateral pulmonary artery banding. After surgery, heart failure worsened with pericardial effusion, pleural effusion, and ascites. In addition, there was prominent trabeculation of the left ventricle and blood signals within the intertrabecular region (Figure 1). Diuretics, inotropic support, and ventilation support were initiated. The pericardial effusion, pleural effusion, and ascites continued for 77 days. The family history was examined, and it revealed that her father and her grandmother had LVNC (Figure 1). The patient is now 2 years old, has severe heart failure, and receives intravenous prostaglandin E1.

Once informed consent from the parents was obtained, blood samples of the patient and parents were taken to perform genetic testing using next-generation sequencing, including 4813 genes (Table 1). A novel heterozygous splicing variant in the $M Y H 7$ gene (c.896$1 \mathrm{G}>\mathrm{A}$ ) was identified in the patient and her father (Figure 2). A heterozygous missense variant in the CD36 gene (c.760T $>$ C, p.Phe254Leu) that was previously reported was identified in the patient and her mother (Figure 2). ${ }^{2}$ Variants were considered likely pathogenic by multiple in silico algorithms (Table 2). The patient and her mother showed reduced CD36 expression compared with the father in both monocytes and platelets (Figure 2).

\section{DISCUSSION}

This is the first fetal case of LVNC associated with variants in $M Y H 7$ and $C D 36$ genes. $M Y H 7$ defects are a major cause of genetic cardiomyopathies, including LVNC. ${ }^{3}$ The variant in $M Y H 7$ (c.896-1G>A) identified in this study affects an amino acid located in the myosin head region, 

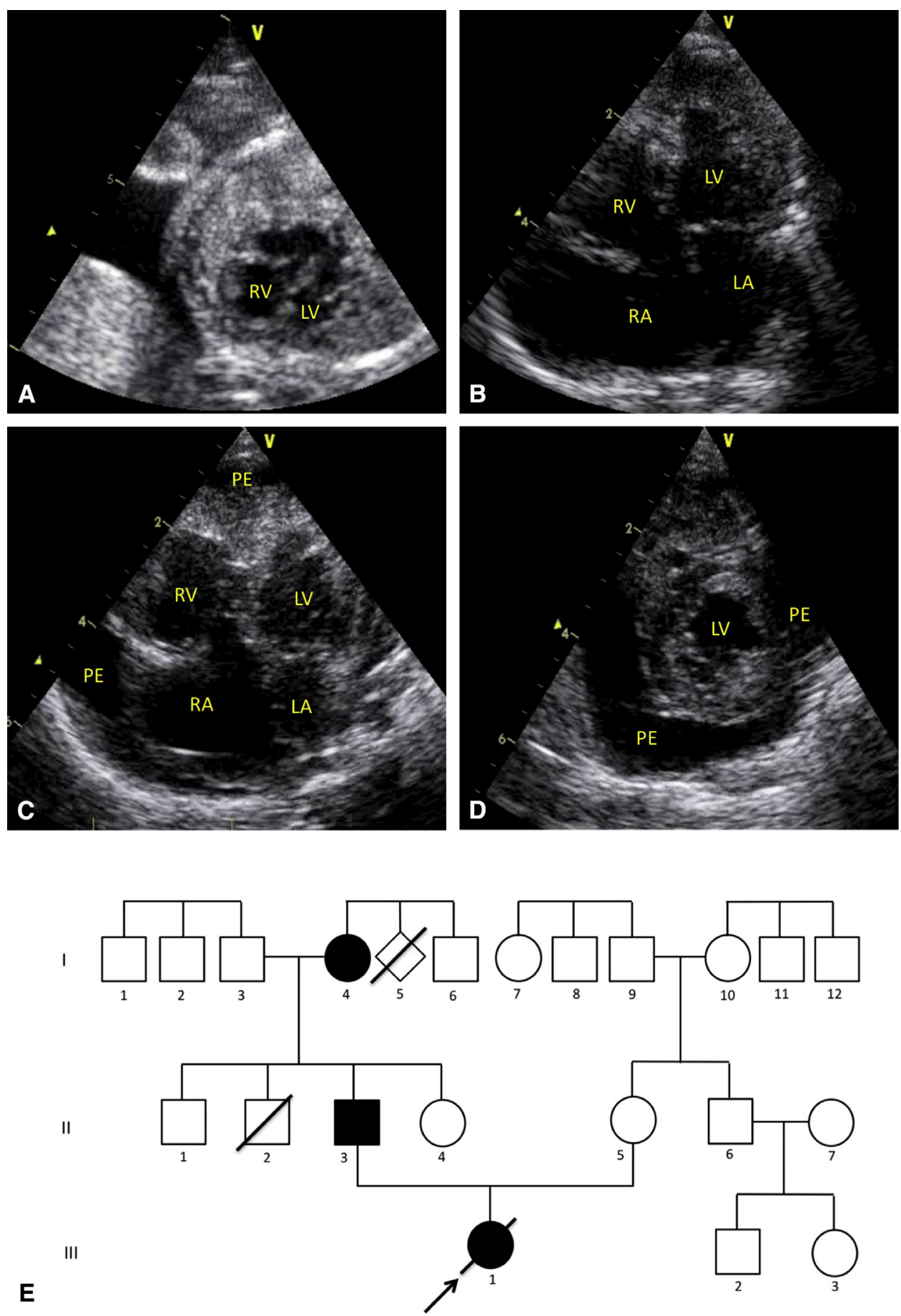

FIGURE 1. Echocardiography image shows an abnormal, highly trabeculated left ventricular myocardium at 30 weeks of gestation (A), at 0 days of age (B), and at 38 days of age in 4-chamber view (C) and short-axis view (D). E, Pedigrees of the patient. Filled symbols indicate individuals with LVNC; open symbols, unaffected status. $R V$, Right ventricle; $L V$, left ventricle; $R A$, right atrium; $L A$, left atrium; $P E$, pericardial effusion.

which contains all the necessary elements to generate movement of actin relative to myosin during ATP hydrolysis. Defective CD36 is a likely candidate gene for cardiomyopathy. ${ }^{2}$ Therefore, we hypothesize that the reason why our case with multiple variants was young at diagnosis and presented with heart failure is that our case 
A2M, A4GALT, A4GNT, AAAS, AADAC, AADACL2, AANAT, AARS, AARS2, AASS, ABAT, ABCA1, ABCA10, ABCA12, ABCA13, ABCA2, ABCA3, ABCA4, $A B C A 7, A B C B 1, A B C B 11, A B C B 4, A B C B 6, A B C B 7, A B C C 1, A B C C 11, A B C C 2, A B C C 3, A B C C 4, A B C C 6, A B C C 8, A B C C 9, A B C D 1, A B C D 3$, ABCD4, ABCG1, ABCG2, ABCG5, ABCG8, ABHD12, ABHD5, ABI3BP, ABO, ACACA, ACACB, ACAD8, ACAD9, ACADL, ACADM, ACADS, ACADSB, ACADVL, ACAN, ACAT1, ACAT2, ACBD5, ACBD6, ACCS, ACE, ACHE, ACLY, ACO2, ACOX1, ACP5, ACSF3, ACSL4, ACSL5, ACSM2B, ACSM3, ACTA1, ACTA2, ACTB, ACTC1, ACTG1, ACTN2, ACTN3, ACTN4, ACVR1, ACVR1B, ACVR1C, ACVR2B, ACVRL1, ACY1, ADA, ADAM10, ADAM12, ADAM17, ADAM19, ADAM33, ADAM7, ADAM9, ADAMTS10, ADAMTS13, ADAMTS16, ADAMTS17, ADAMTS18, ADAMTS2, ADAMTSL2, ADAMTSL3, ADAMTSL4, ADAR, ADARB1, ADC, ADCK3, ADCY10, ADCY3, ADCY5, ADCY6, ADCY9, ADCYAP1, ADD1, ADH1B, ADH1C, ADH4, ADH5, ADH7, ADIPOQ, ADIPOR1, ADK, ADM, ADNP, ADORA1, ADORA2A, ADORA3, ADRA1A, ADRA2A, ADRA2B, ADRA2C, ADRB1, ADRB2, ADRB3, ADRBK2, ADSL, ADTRP, AFF2, AFF3, AFG3L2, AFP, AGA, AGBL4, AGER, AGGF1, AGK, AGL, AGMO, AGO1, AGO2, AGPAT2, AGPS, AGRN, AGRP, AGT, AGTR1, AGTR2, AGXT, AGXT2, AHCY, AHI1, AHR, AHRR, AHSG, AHSP, AICDA, AIF1, AIFM1, AIMP1, AIP, AIPL1, AIRE, AK1, AK2, AKAP10, AKAP13, AKAP2, AKAP9, AKR1B1, AKR1C2, AKR1C3, AKR1C4, AKR1D1, AKR7A2, AKR7A3, AKT1, AKT2, AKT3, ALAD, ALAS2, ALB, ALCAM, ALDH16A1, ALDH18A1, ALDH1A1, ALDH1A2, ALDH2, ALDH3A2, ALDH4A1, ALDH5A1, ALDH6A1, ALDH7A1, ALDOA, ALDOB, ALG1, ALG10B, ALG11, ALG12, ALG13, ALG2, ALG3, ALG6, ALG8, ALG9, ALK, ALMS1, ALOX12, ALOX12B, ALOX15, ALOX5, ALOX5AP, ALOXE3, ALPL, ALS2, ALS2CL, ALX1, ALX3, ALX4, AMACR, AMELX, AMELY, AMER1, AMH, AMHR2, AMN, AMPD1, AMPD3, AMT, ANAPC1, ANG, ANGPT1, ANGPTL3, ANGPTL4, ANGPTL5, ANK1, ANK2, ANK3, ANKH, ANKK1, ANKRD1, ANKRD11, ANKRD26, ANKS1A, ANKS1B, ANO10, ANO5, ANO6, ANO7, ANTXR1, ANTXR2, ANXA11, ANXA5, AOAH, AOC1, AP1S1, AP1S2, AP3B1, AP3B2, AP4B1, AP4E1, AP4M1, AP4S1, AP5Z1, APAF1, APBA2, APBB1, APBB3, APC, APCDD1, APEX1, APH1A, APH1B, APLNR, APOA1, APOA2, APOA4, APOA5, APOB, АPOBEC3B, APOBEC 3G, APOBEC3H, APOC1, APOC2, APOC3, APOC4, APOD, APOE, APOH, APOL1, APOL3, APOM, APP, APRT, APTX, AQP1, AQP2, AQP3, AQP4, AQP5, AQP7, AR, AREL1, ARF4, ARFGEF2, ARG1, ARHGAP24, ARHGAP31, ARHGAP6, ARHGAP9, ARHGEF10, ARHGEF11, ARHGEF12, ARHGEF6, ARHGEF7, ARHGEF9, ARID1A, ARID1B, ARL11, ARL13B, ARL14EP, ARL6, ARL6IP5, ARMS2, ARPC3, ARSA, ARSB, ARSE, ARSF, ART4, ARVCF, ARX, AS3MT, ASAH1, ASAH2, ASB10, ASCC1, ASCC3, ASCL1, ASIC3, ASIP, ASL, ASMT, ASMTL, ASNS, ASPA, ASPM, ASPN, ASPRV1, ASS1, ASTN2, ASXL1, ATCAY, ATF1, ATF3, ATF5, ATF6, ATG16L1, ATG7, ATIC, ATL1, ATM, ATN1, ATOH7, ATP10A, ATP10D, ATP13A2, ATP13A4, ATP1A2, ATP1A3, ATP1B1, ATP2A1, ATP2A2, ATP2A3, ATP2B2, ATP2B4, ATP2C1, ATP5E, ATP5SL, ATP6AP2, ATP6V0A1, ATP6V0A2, ATP6V0A4, ATP6V1B1, ATP7A, ATP7B, ATP8A2, ATP8B1, ATPAF2, ATR, ATRNL1, ATRX, ATXN1, ATXN10, ATXN2, ATXN3, ATXN3L, ATXN7, ATXN8OS, AUH, AURKA, AURKC, AUTS2, AVP, AVPR1A, AVPR1B, AVPR2, AXIN1, AXIN2, AXL, B2M, B3GALNT1, B3GALT6, B3GALTL, B3GAT3, B3GNT1, B3GNT3, B4GALT1, B4GALT7, B9D1, B9D2, BAALC, BAAT, BACE1, BAG3, BAG6, BANF1, BANK1, BAP1, BARD1, BAX, BBS1, BBS10, BBS12, BBS2, BBS4, BBS5, BBS7, BBS9, BCAM, BCAP31, BCAT1, BCAT2, BCHE, BCKDHA, BCKDHB, BCL10, BCL11A, BCL2, BCL2A1, BCL2L11, BCL2L2, BCL9, BCMO1, BCOR, BCORL1, BCR, BCS1L, BDKRB2, BDNF, BEAN1, BEST1, BEX4, BFSP1, BFSP2, BHLHA9, BHLHE41, BHMT, BICC1, BICD1, BIN1, BIRC5, BLK, BLM, BLMH, BLNK, BLOC1S3, BLOC1S6, BLVRA, BMI1, BMP1, BMP10, BMP15, BMP2, BMP2K, BMP4, BMP5, BMP7, BMPER, BMPR1A, BMPR1B, BMPR2, BNC2, BOLA3, BPGM, BPI, BPIFA1, BPY2, BRAF, BRAP, BRAT1, BRCA1, BRCA2, BRCC3, BRD1, BRIP1, BRSK2, BRWD1, BRWD3, BSCL2, BSG, BSND, BST1, BTAF1, BTBD9, BTC, BTD, BTK, BTLA, BTN1A1, BTN2A1, BTNL2, BTRC, BUB1B, C10orf11, C10orf137, C10orf2, C12orf10, C12orf57, C12orf65, C19orf12, C1GALT1, C1QA, C1QB, C1QC, C1QTNF5, C1R, C1S, C2, C2orf71, C3, C3AR1, C4A, C4B, C4BPA, C5, C5AR2, C5orf42, C6, C6orf15, C7, C7orf10, C8A, C8B, C8orf37, C9, C9orf72, CA1, CA12, CA2, CA4, CA6, CA8, CABIN1, CABP4, CACNA1A, CACNA1C, CACNA1D, CACNA1E, CACNA1F, CACNA1G, CACNA1H, CACNA1S, CACNA2D1, CACNA2D3, CACNA2D4, CACNB2, CACNB4, CACNG2, CADM1, CALCA, CALCR, CALHM1, CALM1, CALM3, CALR, CALR3, CAMK4, CAMKK1, CAMKK2, CAMP, CAMTA1, CANT1, CAPN10, CAPN13, CAPN3, CARD14, CARD8, CARD9, CARTPT, CASC16, CASC5, CASK, CASP1, CASP10, CASP12, CASP2, CASP3, CASP5, CASP8, CASP9, CASQ2, CASR, CAST, CAT, CATSPER1, CATSPER2, CATSPER3, CATSPER4, CAV1, CAV3, CBFB, CBL, CBLB, CBR1, CBR3, CBS, CBX2, CBX4, CC2D1A, CC2D2A, CCBE1, CCDC103, CCDC14, CCDC170, CCDC22, CCDC28B, CCDC39, CCDC40, CCDC50, CCDC8, CCDC88C, CCHCR1, CCK, CCKAR, CCKBR, CCL11, CCL13, CCL17, CCL2, CCL22, CCL26, CCL3L1, CCLAL1, CCL5, CCL7, CCM2, CCNA2, CCND1, CCR1, CCR2, CCR3, CCR5, CCR6, CCR7, CCRL2, CCT5, CD109, CD14, CD151, CD177, CD19, CD1A, CD1E, CD207, CD209, CD22, CD226, CD24, CD244, CD247, CD27, CD2AP, CD320, CD36, CD38, CD3D, CD3E, CD3EAP, CD3G, CD4, CD40, CD40LG, CD44, CD46, CD5, CD55, CD58, CD59, CD72, CD79A, CD79B, CD81, CD86, CD8A, CD96, CDA, CDAN1, CDC42BPB, CDC6, CDC73, CDH1, CDH12, CDH13, CDH15, CDH23, CDH3, CDH5, CDH8, CDHR1, CDK11A, CDK4,CDK5R1, CDK5RAP2, CDK5RAP3, CDK7, CDKL3, CDKL5, CDKN1A, CDKN1B, CDKN1C, CDKN2A, CDKN2B, CDKN2B-AS1, CDKN2C, CDON, CDSN, CDT1, CDY1, CDY2A, CEACAM16, CEBPA, CEBPE, CEL, CELSR1, CENPJ, CEP135, CEP152, CEP290, CEP41, CEP57, CEP63, CEP68, CEP85L, CER1, CERKL, CERS6, CES1, CES2, CETP, CFB, CFC1, CFD, CFH, CFHR1, CFHR2, CFHR3, CFHR4, CFHR5, CFI, CFL2, CFLAR, CFP, CFTR, CGA, CGB, CHAT, CHDIL, CHD2, CHD3, CHD6, CHD7, CHD8, CHDH, CHEK2, CHFR, CHGA, CHGB, CHI3L1, CHI3L2, CHIA, CHIT1, CHKB, CHL1, CHM, CHMP2B, CHMP4B, CHN1, CHRDL1, CHRFAM7A, CHRM1, CHRM2, CHRM3, CHRNA1, CHRNA2, CHRNA3, CHRNA4, CHRNA5, CHRNA7, CHRNA9, CHRNB1, CHRNB2, CHRNB4, CHRND, CHRNE, CHRNG, CHST14, CHST3, CHST6, CHST8, CHSY1, CHUK, CIB2, CIC, CIDEA, CIDEC, CIITA, CILP, CIRH1A, CISD2, CISH, CITED2, CKM, CLCA1, CLCA2, CLCF1, CLCN1, CLCN2, CLCN5, CLCN7, CLCNKA, CLCNKB, CLDN1, CLDN14, CLDN16, CLDN19, CLEC11A, CLEC2D, CLEC4M, CLEC7A, CLIC2, CLK2, CLMP, CLN3, CLN5, CLN6, CLN8, CLNK, CLOCK, CLPS, CLPTM1, CLRN1, CLSTN2, CLTCL1, CLU, CLUL1, CLYBL, CMA1, CMPK1, CNBP, CNDP1, CNGA1, CNGA3, CNGB1, CNGB3, CNKSR1, CNKSR2, CNNM2, CNNM4, CNOT3, CNOT4, CNPY3, CNR1, CNR2, CNTF, CNTN1, CNTN4, CNTNAP2, CNTNAP4, CNTNAP5, COA5, COCH, COG1, COG4, COG5, COG6, COG7, COG8, COL10A1, COL11A1, COL11A2, COL12A1, COL17A1, COL18A1, COL1A1, COL1A2, COL25A1, COL2A1, COL3A1, COLAA1, COL4A2, COL4A3, COL4A4, COL4A5, COL4A6, COL5A1, COL5A2, COL6A1, COL6A2, COL6A3, COL6A4P2, COL6A5, COL7A1, COL8A2, COL9A1, COL9A2, COL9A3, COLEC11, COLQ, COMMD1, COMP, COMT, COQ2, COQ4, COQ5, COQ6, COQ9, CORIN, CORO1A, COX10, COX14, COX15, COX4I1, COX412, COX6B1, COX7A1, COX7A2, CP, CPA4, CPA6, CPB2, CPE, CPLX2, CPN1, 
TABLE 1. Continued

CPOX, CPS1, CPT1A, CPT1B, CPT2, CPZ, CR1, CR2, CRADD, CRB1, CRBN, CREB1, CREB3L3, CREBBP, CRELD1, CRH, CRHR1, CRISP2, CRK, CRKL, CRLF1, CRP, CRTAP, CRX, CRYAA, CRYAB, CRYBA1, CRYBA4, CRYBB1, CRYBB2, CRYBB3, CRYGB, CRYGC, CRYGD, CRYGEP, CRYGS, CRYM, CSAG1, CSDE1, CSF1, CSF1R, CSF2, CSF2RA, CSF2RB, CSF3R, CSGALNACT1, CSH1, CSMD1, CSMD3, CSNK1A1L, CSNK1D, CSNK1E, CSNK2A2, CSNK2A3, CSRP3, CST3, CSTA, CSTB, CSTF2T, CTC1, CTDP1, CTF1, CTGF, CTH, CTHRC1, CTLA4, CTNNA3, CTNNB1, CTNND1, CTNS, CTRC, CTSA, CTSB, CTSC, CTSD, CTSG, CTSK, CTSZ, CTTNBP2, CUBN, CUL3, CULAB, CUL5, CUL7, CX3CR1, CXCL10, CXCL11, CXCL12, CXCL16, CXCL5, CXCR1, CXCR3, CXCR4, CYB5A, CYB5R3, CYBA, CYBB, CYBRD1, CYCS, CYLD, CYP11A1, CYP11B1, CYP11B2, CYP17A1, CYP19A1, CYP1A1, CYP1A2, CYP1B1, CYP21A2, CYP24A1, CYP26A1, CYP26B1, CYP27A1, CYP27B1, CYP2A13, CYP2A6, CYP2B6, CYP2C18, CYP2C19, CYP2C8, CYP2C9, CYP2D6, CYP2D7P1, CYP2E1, CYP2F1, CYP2G1P, CYP2J2, CYP2R1, CYP2W1, CYP3A4, CYP3A43, CYP3A5, CYP3A5P1, CYP3A7, CYP46A1, CYP4A11, CYP4A22, CYP4B1, CYP4F12, CYP4F2, CYP4F22, CYP4F3, CYP4V2, CYP7A1, CYP7B1, CYSLTR1, CYSLTR2, D2HGDH, DAG1, DAO, DAOA, DAPK1, DARC, DARS2, DAZ1, DAZ2, DAZ3, DAZL, DBH, DBI, DBT, DCAF13, DCAF17, DCC, DCDC2, DCK, DCLK1, DCLRE1C, DCN, DCP1B, DCTD, DCTN1, DCX, DCXR, DDAH1, DDAH2, DDB2, DDC, DDOST, DDR1, DDR2, DDX11, DDX20, DDX25, DDX39B, DDX3Y, DDX5, DDX53, DDX58, DEAF1, DEC1 , DECR1, DEFB1, DEFB126, DEFB4A, DES, DFNA5, DFNB31, DFNB59, DGAT1, DGCR14, DGCR2, DGCR5, DGCR6, DGCR8, DGKD, DGUOK, DHCR24, DHCR7, DHDDS, DHFR, DHH, DHODH, DHRS4L1, DHX16, DHX36, DHX37, DIABLO, DIAPH1, DIAPH2, DIAPH3, DICER1, DIO1, DIO2, DIP2A, DIP2B, DIP2C, DIRC2, DIS3L2, DISC1, DISP1, DKC1, DKK2, DKK3, DLAT, DLD, DLG3, DLG5, DLGAP2, DLGAP3, DLL1,DLL3, DLX3,DLX5, DLX6,DMBT1,DMC1,DMD, DMGDH, DMP1, DMPK, DMRT1, DMXL1, DNAAF1, DNAAF2, DNAAF3, DNAH11, DNAH5, DNAH9, DNAI1, DNAI2, DNAJA4, DNAJB2, DNAJB6, DNAJC19, DNAJC5, DNAJC6, DNAL1, DNASE1, DNASE1L3, DNASE2, DND1, DNM1, DNM1L, DNM2, DNMT1, DNMT3A, DNMT3B, DNMT3L, DOC2A, DOCK3, DOCK4, DOCK6, DOCK8, DOCK9, DOK7, DOLK, DPAGT1, DPM1, DPM3, DPP10, DPP6, DPY19L2, DPYD, DPYS, DPYSL2, DRD1, DRD2, DRD3, DRD4, DRD5, DROSHA, DRP2, DSC2, DSC3, DSCAM, DSCR8, DSG1, DSG2, DSG4, DSP, DSPP, DST, DTNA, DTNBP1, DUOX2, DUOXA2, DUSP23, DUX4, DXO, DYM, DYNC1H1, DYNC2H1, DYRK1A, DYSF, DYX1C1, DYX1C1-CCPG1, EARS2, EBAG9, EBP, ECE1, ECI1, ECM1, ECM2, ECSIT, EDA, EDA2R, EDAR, EDARADD, EDN1, EDN2, EDN3, EDNRA, EDNRB, EEF1B2, EEF2K, EFCAB5, EFEMP1, EFEMP2, EFHC1, EFHC2, EFNB1, EFR3A, EFTUD2, EGF, EGFR, EGLN1, EGR2, EGR3, EHD2, EHMT1, EHMT2, EIF2AK3, EIF2B1, EIF2B2, EIF2B3, EIF2B4, EIF2B5, EIF3H, EIF4E, EIF4G1, ELAC2, ELANE, ELAVL2, ELF4, ELK1, ELN, ELOVL4, ELP2, ELP4, EMC4, EMD, EME1, EMG1, EMX2, EN2, ENAM, ENG, ENO1, ENO3, ENPP1, ENSA, ENTPD1, EOMES, EP300, EPAS1, EPB41, EPB41L1, EPB42, EPC2, EPCAM, EPHA2, EPHA3, EPHA5, EPHA7, EPHB2, EPHB6, EPHX1, EPHX2, EPM2A, EPO, EPOR, EPX, ERAP1, ERAP2, ERBB2, ERBB3, ERBB4, ERCC1, ERCC2, ERCC3, ERCC4, ERCC5, ERCC6, ERCC8, ERLIN2, ERMAP, ESAM, ESCO2, ESPN, ESR1, ESR2, ESRRB, ESRRG, ETFA, ETFB, ETFDH, ETHE1, ETNPPL, ETS1, EVC, EVC2, EVI5, EXO1, EXO5, EXOC4, EXOSC3, EXT1, EXT2, EXTL3, EYA1, EYA4, EYS, EZH2, F10, F11, F12, F13A1, F13B, F2, F2R, F2RL1, F3, F5, F7, F8, F9, FA2H, FAAH, FAAH2, FABP1, FABP2, FABP3, FABP4, FABP6, FABP7, FADD, FADS2, FAH, FAM120A, FAM126A, FAM134B, FAM161A, FAM205A, FAM20A, FAM20C, FAM58A, FAM83H, FAM8A1, FAM91A1, FANCA, FANCB, FANCC, FANCD2, FANCE, FANCF, FANCG, FANCI, FANCL, FANCM, FARS2, FAS, FASLG, FASN, FASTKD2, FBLIM1, FBLN1, FBLN5, FBN1, FBN2, FBN3, FBP1, FBXL6, FBXO10, FBXO18, FBXO7, FBXW11, FBXW4, FBXW7, FCAR, FCER1A, FCER2, FCGR1A, FCGR2A, FCGR2B, FCGR3A, FCGR3B, FCGRT, FCN2, FCN3, FCRL3, FDFT1, FECH, FEM1A, FEN1, FERMT1, FERMT3, FEV, FEZF2, FFAR1, FFAR4, FGA, FGB, FGD1, FGD3, FGD4, FGF1, FGF10, FGF14, FGF2, FGF20, FGF23, FGF3, FGF8, FGF9, FGFBP1, FGFR1, FGFR2, FGFR3, FGFR4, FGFRL1, FGG, FH, FHL1, FHL2, FIG4, FIGLA, FIP1L1, FKBP10, FKBP14, FKBP5, FKBP6, FKBPL, FKRP, FKTN, FLCN, FLG, FLNA, FLNB, FLNC, FLT1, FLT3, FLT4, FLVCR1, FLVCR2, FMN1, FMN2, FMO1, FMO2, FMO3, FMO4, FMO5, FMO6P, FMR1, FN1, FN3K, FOLH1, FOLR1, FOXA1, FOXA2, FOXA3, FOXC1, FOXC2, FOXD3, FOXE1, FOXE3, FOXF1, FOXF2, FOXG1, FOXH1, FOXI1, FOXL2, FOXN1, FOXP1, FOXP2, FOXP3, FOXRED1, FPGS, FPR1, FPR2, FRA10AC1, FRAS1, FREM1, FREM2, FREM3, FRG1, FRK, FRMD6, FRMD7, FRMPD4, FRY, FRZB, FSCB, FSCN2, FSHB, FSHR, FTCD, FTH1, FTHL17, FTL, FTO, FTSJ1, FUCA1, FURIN, FUS, FUT1, FUT2, FUT3, FUT6, FUT7, FUT8, FUZ, FXN, FXYD2, FXYD6, FYCO1, FZD1, FZD3, FZD4, FZD6, FZD9, G6PC, G6PC2, G6PC3, G6PD, GAA, GAB2, GABBR1, GABRA1, GABRA5, GABRA6, GABRB3, GABRD, GABRG1, GABRG2, GABRG3, GABRR2, GAD1, GAD2, GADD45B, GAK, GAL3ST3, GALC, GALE, GALK1, GALNS, GALNT12, GALNT18, GALNT2, GALNT3, GALNT9, GALNTL5, GALP, GALT, GAMT, GAN, GAP43, GARS, GAS1, GAS2L2, GAS6, GATA1, GATA2, GATA3, GATA4, GATA6, GATAD1, GATM, GBA, GBA3, GBE1, GBGT1, GC, GCDH, GCGR, GCH1, GCK, GCKR, GCLC, GCLM, GCM2, GCNT2, GCSH, GDAP1, GDF1, GDF15, GDF3, GDF5, GDF6, GDF9, GDI1, GDNF, GEMIN2, GEMIN4, GFAP, GFER, GFI1, GFI1B, GFM1, GFPT1, GFPT2, GFRA1, GGCX, GGH, GGT5, GH1, GH2, GHR, GHRH, GHRHR, GHRL, GHSR, GIF, GIGYF2, GIMAP8, GIP, GIPC3, GIPR, GIT1, GJA1, GJA3, GJA4, GJA5, GJA8, GJB1, GJB2, GJB3, GJB4, GJB6, GJC2, GJC3, GJD2, GK, GLA, GLB1, GLCCI1, GLDC, GLE1, GLI1, GLI2, GLI3, GLIS2, GLIS3, GLMN, GLO1, GLP1R, GLRA1, GLRB, GLRX5, GLS, GLTSCR1, GLUD1, GLUD2, GLUL, GLYCTK, GM2A, GMIP, GNA14, GNAI2, GNAQ, GNAS, GNAS-AS1, GNAT1, GNAT2, GNB1L, GNB3, GNB5, GNE, GNMT, GNPAT, GNPTAB, GNPTG, GNRH1, GNRHR, GNS, GOLGA3, GOLGA5, GON4L, GORAB, GOSR2, GOT1, GP1BA, GP1BB, GP2, GP6, GP9, GPAM, GPANK1, GPATCH8, GPBAR1, GPC3, GPC4, GPC6, GPD1, GPDIL, GPD2, GPHN, GPI, GPIHBP1, GPR1, GPR12, GPR139, GPR143, GPR179, GPR33, GPR55, GPR56, GPR68, GPR98, GPS1, GPSM2, GPT, GPX1, GPX4, GRB10, GREM1, GRHL2, GRHPR, GRIA3, GRID1, GRIK1, GRIK2, GRIK3, GRIK4, GRIN1, GRIN2A, GRIN2B, GRIN3A, GRIP1, GRK1, GRK4, GRK5, GRM1, GRM3, GRM5, GRM6, GRM7, GRM8, GRN, GRPR, GRXCR1, GSC, GSDMA, GSDMB, GSE1, GSK3B, GSN, GSPT1, GSPT2, GSR, GSS, GSTA1, GSTA2, GSTA3, GSTK1, GSTM1, GSTM3, GSTM4, GSTO1, GSTO2, GSTP1, GSTT1, GSTT2, GSTT2B, GSTZ1, GTDC2, GTF2E1, GTF2H1, GTF2H5, GTF2IRD1, GTF2IRD2, GUCA1A, GUCA1B, GUCY2C, GUCY2D, GUSB, GYG1, GYLTL1B, GYPA, GYPB, GYPC, GYPE, GYS1, GYS2, GZMB, H19, H2BFWT, H6PD, HABP2, HACE1, HADH, HADHA, HADHB, HAL, HAMP, HAND1, HAND2, HARS, HARS2, HAS1, HAVCR1, HAX1, HBA1, HBA2, HBB, HBD, HBE1, HBEGF, HBG1, HBG2, HBM, HBS1L, HBZ, HCCS, HCK, HCLS1, HCN1, HCN2, HCN4, HCP5, HCRT, HCRTR1, HCRTR2, HDAC4, HDAC8, HDAC9, HDC, HDLBP, HDX, HEATR2, HELQ, HEPACAM, HERC2, HES6, HES7, HESX1, HEXA, HEXB, HEY1, HFE, HFE2, HGD, HGF, HGSNAT, HHEX, HHIP, HIBCH, HIF1A, HIF1AN, HIGD2A, HIP1, HIST1H2AE, HIST3H3, HK1, HK2, HLA-A, HLA-B, HLA-C, HLA-DMB, HLA-DOA, HLA-DPB1, HLA-DPB2, HLA-DQA1, 
HLA-DQB1, HLA-DRA, HLA-DRB1, HLA-DRB5, HLA-E, HLA-G, HLCS, HLX, HMBS, HMCN1, HMGA1, HMGA2, HMGCL, HMGCR, HMGCS2, HMHA1, HMOX1, HMOX2, HMSD, HMX1, HMX2, HNF1A, HNF1B, HNF4A, HNMT, HNRNPH3, HNRNPU, HOGA1, HOMER2, HOXA1, HOXA10, HOXA11, HOXA13, HOXА2, HOXA4, HOXB13, HOXB6, HOXD10, HOXD13, HOXD4, HP, HPD, HPGD, HPRT1, HPS1, HPS3, HPS4, HPS5, HPS6, HPSE2, HR, HRAS, HRC, HRG, HRH2, HRH3, HS1BP3, HS6ST1, HSD11B1, HSD11B2, HSD17B1, HSD17B10, HSD17B2, HSD17B3, HSD17B4, HSD3B1, HSD3B2, HSD3B7, HSF4, HSFY1, HSN2, HSP90AA1, HSP90B1, HSPA1A, HSPA1B, HSPA1L, HSPA5, HSPA8, HSPA9, HSPB1, HSPB3, HSPB6, HSPB7, HSPB8, HSPD1, HSPG2, HTN3, HTR1A, HTR1B, HTR2A, HTR2B, HTR2C, HTR3A, HTR3B, HTR3C, HTR3E, HTR5A, HTR6, HTR7, HTRA1, HTRA2, HTT, HUS1B, HUWE1, HVCN1, HYAL1, HYDIN, HYLS1, HYMAI, IAPP, IBSP, ICAM1, ICAM4, ICAM5, ICK, ICOS, ID3, IDE, IDH1, IDH2, IDH3B, IDO1, IDS, IDUA, IER3IP1, IFI30, IFI44L, IFIH1, IFITM3, IFITM5, IFNA10, IFNA17, IFNA2, IFNAR1, IFNAR2, IFNG, IFNGR1, IFNGR2, IFNL3, IFRD1, IFT122, IFT140, IFT43, IFT80, IGBP1, IGF1, IGF1R, IGF2, IGF2R, IGFALS, IGFBP1, IGFBP3, IGFBP5, IGFBP7, IGHA1, IGHG2, IGHM, IGHMBP2, IGKV, IGLL1, IHH, IKBIP, IKBKAP, IKBKG, IL10, IL10RA, IL10RB, IL11RA, IL12A, IL12B, IL12RB1, IL12RB2, IL13, IL16, IL17A, IL17F, IL17RA, IL17RB, IL17REL, IL18, IL18R1, IL18RAP, IL19, IL1A, IL1B, IL1R1, IL1RAPL1, IL1RL1, IL1RN, IL2, IL20RA, IL20RB, IL21, IL21R, IL23R, IL2RA, IL2RG, IL3, IL31RA, IL36RN, ILA, IL4R, IL5, IL6, IL6R, IL7, IL7R, IL8, IL9, IL9R, ILDR1, ILK, IMMP2L, IMMT, IMPA2, IMPAD1, IMPDH1, IMPDH2, IMPG2, INF2, INHA, INMT, INPP4A, INS, INSIG1, INSIG2, INSL3, INSL6, INSR, INVS, IQCB1, IQGAP1, IQGAP2, IQGAP3, IQSEC2, IRAK1, IRAK3, IRAK4, IRF1, IRF2, IRF4, IRF5, IRF6, IRF7, IRF8, IRGM, IRS1, IRS2, IRS4, IRX4, IRX5, ISCU, ISL1, ISPD, ISYNA1, ITCH, ITGA11, ITGA2, ITGA2B, ITGA3, ITGA4, ITGA6, ITGA7, ITGA9, ITGAE, ITGAM, ITGB2, ITGB3, ITGB4, ITIH1, ITIH3, ITIH4, ITIH6, ITK, ITM2B, ITPA, ITPKC, ITPR1, ITPR3, ITSN2, IVD, IYD, JAG1, JAG2, JAK2, JAK3, JAM3, JMJD1C, JPH2, JPH3, JRK, JUN, JUP, KAL1, KALRN, KANK1, KANSL1, KARS, KAT6B, KATNAL2, KBTBD13, KCNA1, KCNA3, KCNA5, KCNAB2, KCNC3, KCND2, KCND3, KCNE1, KCNE1L, KCNE2, KCNE3, KCNE4, KCNH2, KCNIP4, KCNJ1, KCNJ10, KCNJ11, KCNJ13, KCNJ15, KCNJ2, KCNJ3, KCNJ5, KCNJ6, KCNJ8, KCNJ9, KCNK18, KCNK6, KCNK9, KCNMA1, KCNMB1, KCNMB3, KCNN3, KCNQ1, KCNQ2, KCNQ3, KCNQ4, KCNS1, KCNS3, KCNV2, KCTD13, KCTD7, KDM4C, KDM5A, KDM5C, KDM5D, KDM6A, KDM6B, KDR, KEL, KERA, KHDC3L, KHK, KIAA0100, KIAA0196, KIAA0226, KIAA0232, KIAA0319, KIAA0513, KIAA1033, KIAA1199, KIAA1279, KIAA1377, KIAA1432, KIAA1462, KIAA2022, KIF11, KIF17, KIF18A, KIF1A, KIF1B, KIF21A, KIF22, KIF5A, KIF5B, KIF6, KIF7, KIFAP3, KIR2DL1, KIR2DL3, KIR2DL4, KIR3DL1, KIR3DL2, KIRREL3, KISS1, KISS1R, KIT, KITLG, KL, KLB, KLF1, KLF10, KLF11, KLF5, KLF6, KLF7, KLHDC8B, KLHL10, KLHL3, KLHL7, KLHL9, KLK1, KLK12, KLK15, KLK3, KLK4, KLK7, KLKB1, KLRK1, KMT2C, KMT2D, KMT2E, KNG1, KPNA1, KRAS, KRIT1, KRT1, KRT10, KRT12, KRT13, KRT14, KRT16, KRT17, KRT18, KRT2, KRT3, KRT31, KRT37, KRT38, KRT4, KRT5, KRT6A, KRT6B, KRT6C, KRT74, KRT75, KRT8, KRT81, KRT83, KRT85, KRT86, KRT9, KRTAP1-1, KYNU, L1CAM, L2HGDH, L3MBTL1, LAMA1, LAMA2, LAMA3, LAMA4, LAMA5, LAMB1, LAMB2, LAMB3, LAMC1, LAMC2, LAMC3, LAMP2, LAMTOR2, LARGE, LARS2, LBP, LBR, LCA5, LCAT, LCE3B, LCE3C, LCE5A, LCN10, LCT, LDB3, LDHA, LDHB, LDLR, LDLRAD4, LDLRAP1, LEFTY2, LEMD3, LEP, LEPR, LEPRE1, LEPREL1, LETM1, LFNG, LGALS13, LGALS2, LGALS3, LGI1, LHB, LHCGR, LHFPL5, LHX1, LHX3, LHX4, LHX8, LIAS, LIF, LIFR, LIG1, LIG3, LIG4, LILRA3, LIM2, LIMK1, LIN28A, LIN28B, LINS, LIPA, LIPC, LIPE, LIPG, LIPH, LIPI, LIPN, LITAF, LLGL1, LMAN1, LMBR1, LMBRD1, LMF1, LMNA, LMNB1, LMNB2, LMTK3, LMX1B, LNX2, loc344967, LOR, LOX, LOXHD1, LOXL1, LOXL2, LPA, LPAR1, LPAR6, LPIN1, LPIN2, LPIN3, LPL, LPP, LRAT, LRCH1, LRFN5, LRP1, LRP2, LRP4, LRP5, LRP6, LRP8, LRPAP1, LRPPRC, LRRC4, LRRC6, LRRC8A, LRRK2, LRSAM1, LRTOMT, LTA, LTBP1, LTBP2, LTBP3, LTBP4, LTC4S, LTF, LTK, LTN1, LUM, LY96, LYN, LYST, LYZ, LZTFL1, LZTS1, MACROD2, MAD1L1, MAD2L1, MADD, MAF, MAFB, MAGEE2, MAGEL2, MAGI2, MAGT1, MAK, MAMLD1, MAN1A2, MAN1B1, MAN2B1, MANBA, MAOA, МAOB, MAP2, MAP2K1, MAP2K2, MAP2K3, MAP2K4, MAP3K1, MAP3K15, MAP4K5, MAP6, MAP7D3, MAPK10, MAPK8IP1, MAPT, MARS2, MARVELD2, MASP1, MASP2, MAST4, MASTL, MAT1A, MATN3, MATR3, MAVS, MAX, MBD1, MBD3, MBD4, MBD5, MBL2, MBTPS2, MC1R, MC2R, MC3R, MC4R, MCCC1, MCCC2, MCEE, MCF2L2, MCFD2, MCHR1, MCL1, MCM3AP, MCM4, MCM5, MCM6, MCOLN1, MCPH1, MDH1, MDM2, MDM4, MDN1, MECP2, MED12, MED13, MED13L, MED17, MED23, MED25, MEF2A, MEF2C, MEFV, MEGF10, MEGF11, MEIS1, MEIS2, MEN1, MEP1B, MERTK, MESDC2, MESP2, MEST, MET, MFF, MFGE8, MFI2, MFN2, MFRP, MFSD2A, MFSD8, MGAT1, MGAT2, MGAT4C, MGEA5, MGLL, MGMT, MGP, MGST2, MGST3, MIA3, MIAT, MICA, MICB, MIDI, MIF, MIIP, MINPP1, MIP, MIPOL1, MIR106B, MIR124-1, MIR125A, MIR126, MIR140, MIR146A, MIR16-1, MIR17, MIR17HG, MIR182, MIR184, MIR191, MIR196A2, MIR206, MIR24-1, MIR27A, MIR2861, MIR30C1, MIR34B, MIR499A, MIR502, MIR510, MIR890, MIR892B, MIR934, MIR96, MIRLET7E, MITF, MKKS, MKL1, MKRN3, MKS1, MLC1, MLH1, MLH3, MLLT3, MLPH, MLXIPL, MLYCD, MMAA, MMAB, MMACHC, MMADHC, MME, MMEL1, MMP1, MMP10, MMP12, MMP13, MMP14, MMP2, MMP20, MMP3, MMP7, MMP8, MMP9, MNX1, MOCOS, MOCS1, MOCS2, MOG, MOGS, MOK, MPDU1, MPDZ, MPG, MPHOSPH8, MPI, MPL, MPLKIP, MPO, MPP3, MPP4, MPP6, MPP7, MPST, MPV17, MPZ, MR1, MRAP, MRC1, MRE11A, MRPL3, MRPL48, MRPS16, MRPS22, MRRF, MS4A1, MS4A12, MS4A2, MS4A3, MS4A6A, MS4A6E, MSH2, MSH3, MSH4, MSH5, MSH6, MSMB, MSMO1, MSR1, MSRB3, MST1, MST1R, MSTN, MSX1, MSX2, MT1A, MT2A, MTAP, MTCH2, MTFMT, MTHFD1, MTHFD1L, MTHFR, MTHFS, MTM1, MTMR14, MTMR2, MTMR9, MTNR1A, MTNR1B, MTO1, MTPAP, MTR, MTRR, MTTP, MTUS1, MUC1, MUC13, MUC2, MUC3A, MUC4, MUC5B, MUC6, MUC7, MURC, MUSK, MUT, MUTYH, MVK, MX1, MYB, MYBL2, MYBPC1, MYBPC3, MYC, MYCL, MYCN, MYD88, MYF6, MYH11, MYH13, MYH14, MYH15, MYH2, MYH3, MYH6, MYH7, MYH8, MYH9, MYL2, MYL3, MYLIP, MYLK, MYLK2, MYO15A, MYO18B,

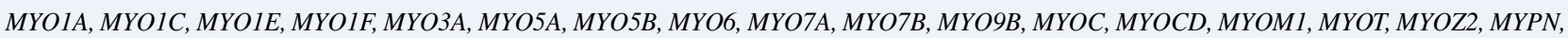
MYT1, MYT1L, NAA10, NAGA, NAGLU, NAGPA, NAGS, NAIP, NAMPT, NAPRT1, NARS2, NAT1, NAT2, NAT8L, NAV2, NBAS, NBEA, NBEAL2, NBN, NBPF1, NCALD, NCAM1, NCAN, NCAPD2, NCF1, NCF2, NCF4, NCKAP1, NCOA1, NCOA3, NCR3, NCS1, NCSTN, NDE1, NDN, NDOR1, NDP, NDRG1, NDST1, NDUFA1, NDUFA10, NDUFA11, NDUFA12, NDUFA13, NDUFA2, NDUFA4, NDUFA6, NDUFA7, NDUFA8, NDUFA9, NDUFAF1, NDUFAF2, NDUFAF3, NDUFAF4, NDUFAF5, NDUFAF6, NDUFAF7, NDUFB1, NDUFB3, NDUFB6, NDUFB9, NDUFC2, NDUFS1, NDUFS2, NDUFS3, NDUFS4, NDUFS5, NDUFS6, NDUFS7, NDUFS8, NDUFV1, NDUFV2, NDUFV3, NEB, NEBL, NEDD4L, NEDD9, NEFH, NEFL, NEFM, NEGR1, NEIL1, NEIL2, NEK1, NEK8, NELFA, NELL1, NEU1, NEU2, NEUROD1, NEUROG3, NEXN, NF1, NF2, NFATC4, NFE2L2, NFIA, NFIX,

(Continued) 
TABLE 1. Continued

NFKB1, NFKBIA, NFKBIL1, NFU1, NGF, NGFR, NHEJ1, NHLRC1, NHP2, NHS, NID1, NINJ1, NIPA1, NIPAL4, NIPBL, NIPSNAP1, NIPSNAP3A, NKAIN2, NKX2-1, NKX2-3, NKX2-5, NKX2-6, NKX3-1, NKX3-2, NLGN1, NLGN2, NLGN3, NLGN4X, NLGN4Y, NLRP1, NLRP12, NLRP14, NLRP2, NLRP3, NLRP7, NLRX1, NMB, NME1, NME8, NMNAT1, NMT2, NMU, NOBOX, NOD1, NOD2, NODAL, NOG, NOP10, NOP56, NOS1, NOS1AP, NOS2, NOS3, NOTCH1, NOTCH2, NOTCH3, NOTCH4, NPAP1, NPAS2, NPAS3, NPAT, NPC1, NPC1L1, NPC2, NPFFR2, NPHP1, NPHP3, NPHP4, NPHS1, NPHS2, NPL, NPM1, NPPA, NPPB, NPPC, NPR1, NPR2, NPR3, NPSR1, NPTN, NPY, NPY1R, NPY2R, NQO1, NQO2, NROB1, NROB2, NR1H2, NR1H3, NR1H4, NR1I2, NR113, NR2E1, NR2E3, NR2F1, NR3C1, NR3C2, NR4A2, NR5A1, NRAS, NRG1, NRG3, NRIP1, NRL, NRP2, NRTN, NRXN1, NRXN2, NRXN3, NSD1, NSDHL, NSMF, NSUN2, NSUN7, NT5C1B, NT5C3A, NT5E, NTF3, NTF4, NTHL1, NTNG1, NTRK1, NTRK2, NTRK3, NUAK1, NUBPL, NUDC, NUDT1, NUDT6, NUMBL, NUP155, NUP62, NXF3, NXF5, NXNL1, NYX, OAS1, OAS2, OAT, OAZ1, OBSCN, OBSL1, OCA2, OCLN, OCRL, ODC1, OFD1, OGG1, OLFM2, OLR1, OMG, OPA1, OPA3, OPCML, OPHN1, OPN1LW, OPN1MW, OPN1SW, OPN4, OPRD1, OPRK1, OPRL1, OPRM1, OPTC, OPTN, OR13G1, OR7D4, ORAI1, ORC1, ORC4, ORC6, ORMDL3, OSMR, OSR1, OSTM1, OTC, OTOA, OTOF, OTOR, OTX2, OVGP1, OXCT1, OXTR, P2RX1, P2RX4, P2RX5, P2RX7, P2RY11, P2RY12, P2RY4, PABPC4L, PABPN1, PACRG, PAFAH1B1, PAFAH1B3, PAH, PAK3, PAK7, PALB2, PALLD, PANK2, PAPD7, PAPSS2, PARD3B, PARD6A, PARK2, PARK7, PARL, PARP1, PARP2, PASK, PAWR, PAX1, PAX2, PAX3, PAX4, PAX5, PAX6, PAX7, PAX8, PAX9, PC, PCBD1, PCCA, PCCB, PCDH11X, PCDH15, PCDH18, PCDH19, PCDH9, PCDHA10, PCDHA13, PCDHB4, PCK1, PCK2, PCM1, PCMT1, PCNT, PCOLCE, PCSK1, PCSK2, PCSK9, PDCD1, PDCD10, PDCD5, PDE10A, PDE11A, PDE4B, PDE4D, PDE6A, PDE6B, PDE6C, PDE6G, PDE6H, PDE7B, PDE8B, PDGFB, PDGFC, PDGFRA, PDHA1, PDHB, PDHX, PDLIM3, PDLIM5, PDP1, PDPK1, PDSS1, PDSS2, PDX1, PDXK, PDYN, PDZD7, PEAR1, PECAM1, PECR, PEMT, PENK, PEPD, PER1, PER2, PER3, PEX1, PEX10, PEX11B, PEX12, PEX13, PEX14, PEX16, PEX19, PEX2, PEX26, PEX3, PEX5, PEX6, PEX7, PFAS, PFKM, PFN1, PGAM1, PGAM2, PGAM5, PGBD1, PGC, PGD, PGK1, PGM1, PGR, PGRMC1, PHB, PHEX, PHF11, PHF2, PHF3, PHF6, PHF8, PHGDH, PHIP, PHKA1, PHKA2, PHKB, PHKG2, PHLDA2, PHLPP2, PHOX2A, PHOX2B, PHYH, PI3, PICALM, PICK1, PIEZO2, PIF1, PIGA, PIGL, PIGM, PIGN, PIGO, PIGR, PIGV, PIK3C2G, PIK3C3, PIK3CA, PIK3CB, PIK3CD, PIK3CG, PIK3R1, PIK3R2, PIK3R4, PIK3R5, PIKFYVE, PIN1, PINK1, PIP4K2A, PIP5K1B, PIP5K1C, PITPNM3, PITX1, PITX2, PITX3, PIWIL3, PKD1, PKD1L1, PKD2, PKHD1, PKLR, PKM, PKN3, PKP1, PKP2, PLA2G10, PLA2G2A, PLA2G2D, PLA2G4A, PLA2G4C, PLA2G5, PLA2G6, PLA2G7, PLAGL1, PLAT, PLAU, PLAUR, PLCB1, PLCB4, PLCD1, PLCE1, PLCG2, PLCZ1, PLD2, PLEC, PLEKHG4, PLEKHG5, PLEKHM1, PLG, PLIN1, PLIN4, PLN, PLOD1, PLOD2, PLOD3, PLP1, PLP2, PLTP, PLXND1, PMAIP1, PML, PMM2, PMP22, PMPCA, PMS1, PMS2, PMS2P3, PNKD, PNKP, PNMT, PNP, PNPLA1, PNPLA2, PNPLA3, PNPLA6, PNPO, POF1B, POGZ, POLB, POLD1, POLE2, POLG, POLG2, POLH, POLL, POLR1C, POLR1D, POLR2E, POLR2M, POLR3A, POLR3B, POLRMT, POMC, POMGNT1, POMP, POMT1, POMT2, PON1, PON2, PON3, POP1, POR, PORCN, POU1F1, POU3F4, POU4F3, POU5F1, POU5F1B, POU6F2, PPARA, PPARD, PPARG, PPARGC1A, PPARGC1B, PPAT, PPIA, PPIB, PPIG, PPM1D, PPOX, PPP1R1A, PPP1R3A, PPP1R3C, PPP2R1A, PPP2R1B, PPP2R2B, PPP2R2C, PPT1, PQBP1, PRB1, PRB3, PRB4, PRCD, PRCP, PRDM2, PRDM5, PRDM9, PREPL, PRF1, PRG4, PRICKLE1, PRICKLE2, PRKAA2, PRKACA, PRKAG2, PRKAG3, PRKAR1A, PRKCB, PRKCG, PRKCH, PRKCSH, PRKD3, PRKDC, PRKRA, PRL, PRLHR, PRLR, PRM1, PRM2, PRMT10, PRMT3, PRMT7, PRND, PRNP, PROC, PROCR, PRODH, PROK1, PROK2, PROKR1, PROKR2, PROM1, PROP1, PROS1, PROZ, PRPF3, PRPF31, PRPF6, PRPF8, PRPH, PRPH2, PRPS1, PRRC2A, PRRT2, PRRX1, PRSS1, PRSS12, PRSS2, PRSS3P2, PRSS56, PRSS8, PRX, PRY, PRY2, PSAP, PSAT1, PSCA, PSEN1, PSEN2, PSENEN, PSMA6, PSMB8, PSMB9, PSMC3IP, PSMD7, PSPH, PSPN, PSTPIP1, PTAFR, PTCD1, PTCH1, PTCH2, PTCHD1, PTCHD3, PTCSC3, PTEN, PTF1A, PTGDR, PTGDR2, PTGDS, PTGER2, PTGER4, PTGES2, PTGIR, PTGIS, PTGS1, PTGS2, PTH, PTH1R, PTHLH, PTK7, PTPN1, PTPN11, PTPN13, PTPN14, PTPN2, PTPN21, PTPN22, PTPN6, PTPRB, PTPRC, PTPRCAP, PTPRD, PTPRF, PTPRJ, PTPRK, PTPRO, PTPRQ, PTPRT, PTRF, PTS, PUS1, PVR, PVRL1, PVRL3, PVRLA, PVT1, PWRN1, PXDN, PYCR1, PYCRL, PYGL, PYGM, PYY, PZP, QDPR, QKI, RAB11FIP5, RAB18, RAB23, RAB27A, RAB28, RAB2A, RAB39B, RAB3GAP1, RAB3GAP2, RAB40AL, RAB7A, RAB7L1, RABGGTA, RABL6, RAC1, RAC2, RAD21, RAD21L1, RAD23B, RAD50, RAD51, RAD51C, RAD51D, RAD52, RAD54B, RAD54L, RAD9A, RAET1L, RAF1, RAG1, RAG2, RAI1, RALGAPA1, RALGDS, RANBP2, RANGRF, RAPSN, RARS2, RASA1, RASGRP2, RASSF1, RAX, RAX2, RB1, RB1CC1, RBBP8, RBFOX1, RBM10, RBM15, RBM20, RBM28, RBM8A, RBMXL2, RBMY1A1, RBP3, RBP4, RCAN1, RD3, RDH12, RDH5, RDX, RECQL4, REEP1, RELN, REN, REPS2, RET, RETN, REV3L, RFC2, RFT1, RFWD2, RFX2, RFX5, RFX6, RFX8, RFXANK, RFXAP, RGMA, RGR, RGS2, RGS6, RGS7, RGS9, RGS9BP, RHAG, RHBDF2, RHCE, RHD, RHO, RHOB, RHOG, RHPN2, RIMS1, RIMS3, RIN2, RIPK3, RIPK4, RLBP1, RMI1, RMRP, RNASE3, RNASEH2A, RNASEH2B, RNASEH2C, RNASEL, RNASET2, RNF113A, RNF114, RNF135, RNF139, RNF168, RNF170, RNF213, RNLS, RNU4ATAC, ROBO1, ROBO2, ROBO3, ROCK1, ROCK2, ROGDI, ROM1, ROPN1L, ROR2, RORA, ROS1, RP1, RP1L1, RP2, RP9, RPA1, RPA4, RPE65, RPGR, RPGRIP1, RPGRIP1L, RPH3AL, RPIA, RPL10, RPL11, RPL21, RPL35A, RPL36, RPL5, RPN2, RPS10, RPS15, RPS17, RPS19, RPS24, RPS26, RPS27A, RPS3, RPS4Y2, RPS6KA3, RPS6KB1, RPS6KL1, RPS7, RPTOR, RRH, RRM1, RRM2B, RRP1B, RS1, RSPH4A, RSPH9, RSPO1, RSPO4, RSRC1, RTN2, RTN4R, RUNX1, RUNX2, RUNX3, RUVBL1, RXFP2, RXRG, RYK, RYR1, RYR2, RYR3, S100A14, S100B, S1PR1, SAA1, SAA2, SACS, SAG, SAGE1, SALL1, SALL4, SAMD9, SAMHD1, SAR1B, SARDH, SARS2, SART1, SART3, SAT1, SATB2, SATL1, SBDS, SBF2, SBNO1, SC5DL, SCAP, SCARB1, SCARB2, SCARF2, SCG2, SCG3, SCGB1A1, SCGB1D2, SCGB3A2, SCN10A, SCN1A, SCN1B, SCN2A, SCN2B, SCN3A, SCN3B, SCN4A, SCN4B, SCN5A, SCN8A, SCN9A, SCNN1A, SCNN1B, SCNN1G, SCO1, SCO2, SCP2, SCRIB, SCT, SCUBE2, SDC3, SDCCAG8, SDHA, SDHAF1, SDHAF2, SDHB, SDHC, SDHD, SEC23A, SEC23B, SEC63, SECISBP2, SEL1L, SELE, SELL, SELP, SELPLG, SEMA3A, SEMA3E, SEMA4A, SEMA4C, SEMA4G, SEMA6D, SEMG1, SEP15, SEPN1, SEPP1, SEPSECS, SEPT12, SEPT9, SERPINA1, SERPINA10, SERPINA3, SERPINA6, SERPINA7, SERPINB11, SERPINB3, SERPINB5, SERPINB6, SERPINC1, SERPIND1, SERPINE1, SERPINF1, SERPINF2, SERPING1, SERPINH1, SERPINI1, SERPINI2, SERTAD1, SESN2, SETBP1, SETD2, SETD8, SETDB2, SETX, SEZ6, SEZ6L2, SF3B4, SFTPA1, SFTPA2, SFTPB, SFTPC, SFTPD, SGCA, SGCB, SGCD, SGCE, SGCG, SGK1, SGK110, SGK223, SGSH, SH2B1, SH2B3, SH2D1A, SH3BP2, SH3GL1, SH3PXD2B, SH3TC2, SHANK2, SHANK3, SHBG, SHFM1, SHH, SHMT1, SHOC2, SHOX, SHROOM3, SHROOM4, SI, SIAE, SIGLEC12, SIGLEC14, SIGLEC16, SIGMAR1, SIL1, SIM1, SIPA1, SIRT1, SIRT3, SIRT5, SIX1, SIX2, SIX3, SIX5, SIX6, SKI, SKIV2L, SLBP, SLC10A1,

(Continued) 
SLC10A2, SLC11A1, SLC11A2, SLC12A1, SLC12A3, SLC12A6, SLC13A2, SLC14A1, SLC14A2, SLC15A1, SLC16A1, SLC16A12, SLC16A2, SLC16A3, SLC17A1, SLC17A3, SLC17A5, SLC17A8, SLC18A1, SLC19A1, SLC19A2, SLC19A3, SLC1A1, SLC1A2, SLC1A3, SLC20A2, SLC22A1, SLC22A11, SLC22A12, SLC22A14, SLC22A2, SLC22A3, SLC22A4, SLC22A5, SLC22A6, SLC22A9, SLC23A1, SLC24A1, SLC24A2, SLC24A5, SLC25A12, SLC25A13, SLC25A15, SLC25A19, SLC25A20, SLC25A22, SLC25A3, SLC25A38, SLC25A39, SLC25A4, SLC26A1, SLC26A10, SLC26A2, SLC26A3, SLC26A4, SLC26A5, SLC26A6, SLC26A9, SLC27A4, SLC27A5, SLC28A1, SLC28A2, SLC28A3, SLC29A1, SLC29A2, SLC29A3, SLC29A4, SLC2A1, SLC2A10, SLC2A2, SLC2A4, SLC2A9, SLC30A10, SLC30A2, SLC30A5, SLC30A8, SLC31A1, SLC33A1, SLC34A1, SLC34A2, SLC34A3, SLC35A1, SLC35C1, SLC35D1, SLC35G2, SLC36A2, SLC37A4, SLC39A13, SLC39A4, SLC3A1, SLC40A1, SLC41A1, SLC44A2, SLC45A2, SLC46A1, SLC47A1, SLC47A2, SLC4A1, SLC4A10, SLC4A11, SLC4A3, SLC4A4, SLC52A1, SLC52A3, SLC5A1, SLC5A11, SLC5A2, SLC5A5, SLC5A7, SLC6A1, SLC6A11, SLC6A12, SLC6A13, SLC6A14, SLC6A18, SLC6A19, SLC6A2, SLC6A20, SLC6A3, SLC6A4, SLC6A5, SLC6A8, SLC7A1, SLC7A10, SLC7A2, SLC7A5, SLC7A7, SLC7A9, SLC8A1, SLC9A3R1, SLC9A6, SLC9A9, SLCO1A2, SLCO1B1, SLCO1B3, SLCO1C1, SLCO2A1, SLCO2B1, SLFN5, SLIT3, SLITRK1, SLURP1, SLX4, SMAD1, SMAD2, SMAD3, SMAD4, SMAD6, SMAD7, SMAD9, SMARCA2, SMARCA4, SMARCAD1, SMARCAL1, SMARCB1, SMARCE1, SMC1A, SMC1B, SMC3, SMG1, SMG6, SMN1, SMN2, SMNDC1, SMOC1, SMOC2, SMPD1, SMPX, SMS, SMUG1, SMYD3, SNAI2, SNAP29, SNAPC4, SNAPC5, SNCA, SNCAIP, SNCB, SNIP1, SNORD115-1, SNORD116-10, SNORD116-1, SNORD50A, SNRK, SNRNP200, SNRPN, SNTA1, SNTG2, SNURF, SNX10, SNX19, SNX3, SOBP, SOCS1, SOCS3, SOD1, SOD2, SOD3, SOGA3, SOHLH1, SORBS1, SORCS1, SORL1, SORT1, SOS1, SOST, SOX10, SOX17, SOX18, SOX2, SOX3, SOX5, SOX6, SOX8, SOX9, SP100, SP110, SP7, SP8, SPAG16, SPAG17, SPANXN5, SPAST, SPATA13, SPATA16, SPATA21, SPATA31C1, SPATA7, SPECC1L, SPG11, SPG20, SPG21, SPG7, SPI1, SPINK1, SPINK5, SPINT2, SPP1, SPR, SPRED1, SPRN, SPRR3, SPRY2, SPRY3, SPTA1, SPTAN1, SPTB, SPTBN2, SPTBN5, SPTLC1, SPTLC2, SQSTM1, SRC, SRCAP, SRD5A2, SRD5A3, SREBF1, SREBF2, SREK1, SRGAP2, SRGAP3, SRI, SRP72, SRPX, SRPX2, SRR, SRY, SSH1, SST, SSTR2, SSTR5, SSX7, ST14, ST3GAL3, ST3GAL5, ST5, ST7, ST8SIA2, STAR, STAT1, STAT3, STAT5B, STAT6, STEAP3, STEAP4, STH, STIL, STIM1, STK10, STK11, STK19, STK3, STK32A, STK33, STK39, STK4, STMN1, STOX1, STRA6, STRADA, STRC, STS, STX11, STX16, STX1A, STXBP1, STXBP2, STXBP5, SUCLA2, SUCLG1, SUFU, SULF1, SULT1A1, SULT1A3, SULT1C2, SULT1E1, SULT2A1, SULT2B1, SULT4A1, SUMF1, SUMO1, SUMO4, SUN2, SUOX, SUPT16H, SURF1, SUV42OH1, SV2B, SYCP3, SYN1, SYN3, SYNE1, SYNE2, SYNGAP1, SYNGR1, SYNM, SYNPO, SYP, SYT11, SYT14, SYT2, SYTL3, SYTL5, T, TAAR6, TAAR9, TAB2, TAC3, TACO1, TACR3, TACSTD2, TAF1, TAF15, TAF1C, TAF1L, TAF2, TAF7L, TAL1, TALDO1, TAP1, TAP2, TAPBP, TARDBP, TAS1R1, TAS1R2, TAS1R3, TAS2R16, TAS2R19, TAS2R3, TAS2R38, TAS2R43, TAS2R46, TAS2R50, TAS2R9, TAT, TAZ, TBC1D1, TBC1D23, TBC1D24, TBC1D4, TBCE, TBK1, TBL1X, TBL1XR1, TBL1Y, TBP, TBX1, TBX10, TBX15, TBX19, TBX2, TBX20, TBX21, TBX22, TBX3, TBX4, TBX5, TBX6, TBXA2R, TBXAS1, TCAP, TCF21, TCF4, TCF7, TCF7L1, TCF7L2, TCIRG1, TCN1, TCN2, TCOF1, TCP1, TCTE1, TCTE3, TCTN1, TCTN2, TDGF1, TDO2, TDP1, TDRD7, TEAD1, TECR, TECTA, TEK, TEKT2, TENM4, TEP1, TERC, TERT, TET1, TEX14, TF, TFAM, TFAP2A, TFAP2B, TFB1M, TFCP2, TFF1, TFPI, TFR2, TFRC, TG, TGFB1, TGFB2, TGFB3, TGFBI, TGFBR1, TGFBR2, TGFBR3, TGFBRAP1, TGIF1, TGM1, TGM2, TGM5, TGM6, TH, THADA, THAP1, THBD, THBS1, THBS2, THBS4, THPO, THRA, THRB, THSD7A, TICAM1, TIMM44, TIMM8A, TIMP1, TIMP2, TIMP3, TINAG, TINF2, TIRAP, TJP2, TK2, TLK1, TLL1, TLR1, TLR10, TLR2, TLR3, TLR4, TLR5, TLR6, TLR7, TLR8, TLR9, TM4SF19, TMC1, TMC6, TMC8, TMCO1, TMEM114, TMEM126A, TMEM127, TMEM135, TMEM138, TMEM165, TMEM173, TMEM185A, TMEM187, TMEM2, TMEM216, TMEM237, TMEM39A, TMEM43, TMEM67, TMEM70, TMEM8A, TMEM9, TMIE, TMLHE, TMPO, TMPRSS11A, TMPRSS15, TMPRSS3, TMPRSS4, TMPRSS5, TMPRSS6, TNC, TNF, TNFAIP2, TNFAIP3, TNFRSF10A, TNFRSF10B, TNFRSF11A, TNFRSF11B, TNFRSF13B, TNFRSF13C,

TNFRSF1A, TNFRSF1B, TNFRSF25, TNFRSF4, TNFSF10, TNFSF11, TNFSF13B, TNFSF14, TNFSF15, TNFSF4, TNFSF8, TNKS, TNNC1, TNNI2, TNNI3, TNNI3K, TNNT1, TNNT2, TNNT3, TNP1, TNR, TNS3, TNXB, TOMM40, TOP1MT, TOPBP1, TOPORS, TOR1A, TOX3, TP53, TP53AIP1, TP53BP1, TP5313, TP53RK, TP63, TP73, TPCN2, TPH1, TPH2, TPI1, TPK1, TPM1, TPM2, TPM3, TPMT, TPO, TPP1, TPP2, TPRN, TPSB2, TPTE, TRAC, TRADD, TRAF3, TRAF3IP1, TRAF3IP2, TRAF6, TRAK2, TRAPPC10, TRAPPC2, TRAPPC9, TRB, TRBV9, TRDN, TREM2, TRERF1, TREX1, TRHR, TRIB1, TRIB3, TRIL, TRIM17, TRIM21, TRIM22, TRIM32, TRIM37, TRIM5, TRIO, TRIOBP, TRIP11, TRIP12, TRMT1, TRMU, TRD, TROAP, TROVE2, TRPA1, TRPC3, TRPC4, TRPC5, TRPC6, TRPM1, TRPM2, TRPM3, TRPM4, TRPM6, TRPM7, TRPS1, TRPV1, TRPV3, TRPV4, TRPV5, TRRAP, TSC1, TSC2, TSEN2, TSEN34, TSEN54, TSFM, TSHB, TSHR, TSHZ1, TSLP, TSPAN12, TSPAN17, TSPAN7, TSPO, TSPYL1, TSSC4, TSSK2, TSSK3, TSSK4, TST, TTBK2, TTC19, TTC21B, TTC37, TTC8, TTI2, TTLL11, TTN, TTPA, TTR, TTTY13, TUBA1A, TUBA8, TUBB1, TUBB2B, TUBB3, TUBGCP4, TUBGCP5, TUBGCP6, TUFM, TULP1, TULP3, TUSC3, TWIST1, TWIST2, TXN2, TXNRD2, TYK2, TYMP, TYMS, TYR, TYROBP, TYRP1, UBA1, UBA3, UBAC2, UBD, UBE2A, UBE2B, UBE2I, UBE2NL, UBE3A, UBE3C, UBIAD1, UBN2, UBQLN2, UBR1, UBR3, UBR7, UCHL1, UCP1, UCP2, UCP3, UFD1L, UGT1A1, UGT1A10, UGT1A3, UGT1A4, UGT1A5, UGT1A6, UGT1A7, UGT1A8, UGT1A9, UGT2A1, UGT2A3, UGT2B10, UGT2B15, UGT2B17, UGT2B28, UGT2B7, UHRF1BP1, UIMC1, UMOD, UMPS, UNC119, UNC13D, UNC5C, UNC5CL, UNC80, UNC93A, UNC93B1, UNG, UNKL, UPB1, UPF3B, UPK3A, UQCRB, UQCRQ, UROC1, UROD, UROS, USB1, USF1, USH1C, USH1G, USH2A, USP15, USP24, USP26, USP46, USP7, USP9X, USP9Y, UTF1, UTRN, UTS2, UVSSA, VAMP7, VANGL1, VANGL2, VAPB, VAX1, VCAM1, VCAN, VCL, VCP, VCX3A, VCY, VDR, VEGFA, VHL, VIM, VIMP, VIP, VIPAS39, VIPR2, VKORC1, VLDLR, VMA21, VNN1, VPREB1, VPS13A, VPS13B, VPS33B, VPS35, VRK1, VSIG4, VSX1, VSX2, VTN, VWF, WAS, WASF3, WDFY3, WDFY4, WDPCP, WDR11, WDR13, WDR19, WDR35, WDR36, WDR4, WDR45, WDR45B, WDR62, WDR65, WDR72, WDR81, WFS1, WHSC1, WIPF1, WISP3, WNK1, WNK4, WNT10A, WNT10B, WNT3, WNT4, WNT5A, WNT5B, WNT7A, WRAP53, WRN, WT1, WT1-AS, WWC1, WWOX, XBP1, XDH, XIAP, XIST, XK, XKR4, XKRY, XPA, XPC, XPNPEP2, XPNPEP3, $X R C C 1, X R C C 2, X R C C 3, X R C C 4, X R C C 5, X R C C 6, X Y L T 1, X Y L T 2, Y A P 1, Y A R S, Y A R S 2, Y B X 2, Y T H D F 2, Y W H A E, Y Y 1, Z A N, Z A P 70, Z B T B 16$, ZBTB18, ZBTB24, ZBTB40, ZBTB41, ZC3H14, ZC3H3, ZC3HAV1, ZCCHC12, ZCCHC13, ZCCHC8, ZDHHC15, ZDHHC8, ZDHHC9, ZEB1, ZEB2, ZFAT, ZFHX3, ZFHX4, ZFP36, ZFP36L1, ZFP57, ZFP90, ZFPM2, ZFY, ZFYVE26, ZFYVE27, ZHX3, ZIC1, ZIC2, ZIC3, ZMPSTE24, ZMYND11, ZNF202, ZNF213, ZNF224, ZNF24, ZNF335, ZNF350, ZNF365, ZNF385B, ZNF41, ZNF469, ZNF480, ZNF507, ZNF513, ZNF526, ZNF592, ZNF627, ZNF644, ZNF674, ZNF711, ZNF750, ZNF75D, ZNF80, ZNF804A, ZNF81, ZNHIT6, ZNRF1, ZPBP, ZPBP2

NGS, Next-generation sequencing. 


\section{MYH7 gene}

Proband
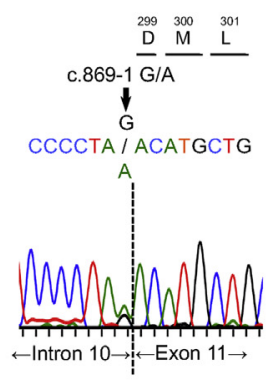

Father
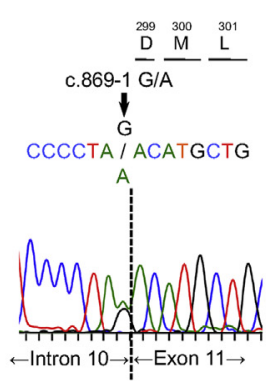

Mother

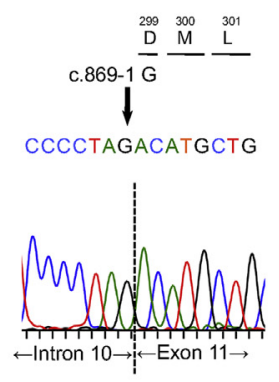

Mother
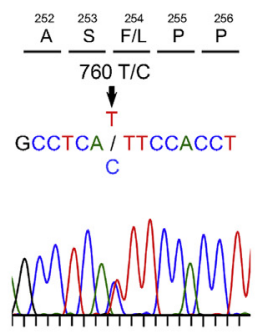

A
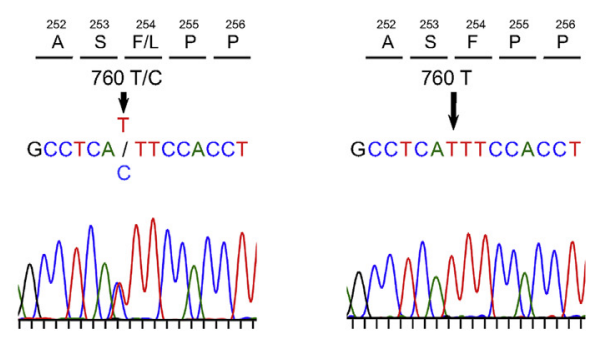

FIGURE 2. A, Sanger sequence analysis of the patient. Flow cytometry analysis for surface expression of CD36 in monocytes (B) and platelets (C) from the patient and parents.

could be caused by a novel variant in the $M Y H 7$ gene, and the heart failure, which worsened after surgical intervention, could be caused by the CD36 abnormality.

In conclusion, our data support the hypothesis that the presence of multiple variants can affect the clinical phenotype and prognosis in LVNC. Our case indicates that the evaluation of cardiac function, the presence of additional congenital malformations and family history, along with identification of the molecular defect, are likely to be important before and after cardiac surgery. 


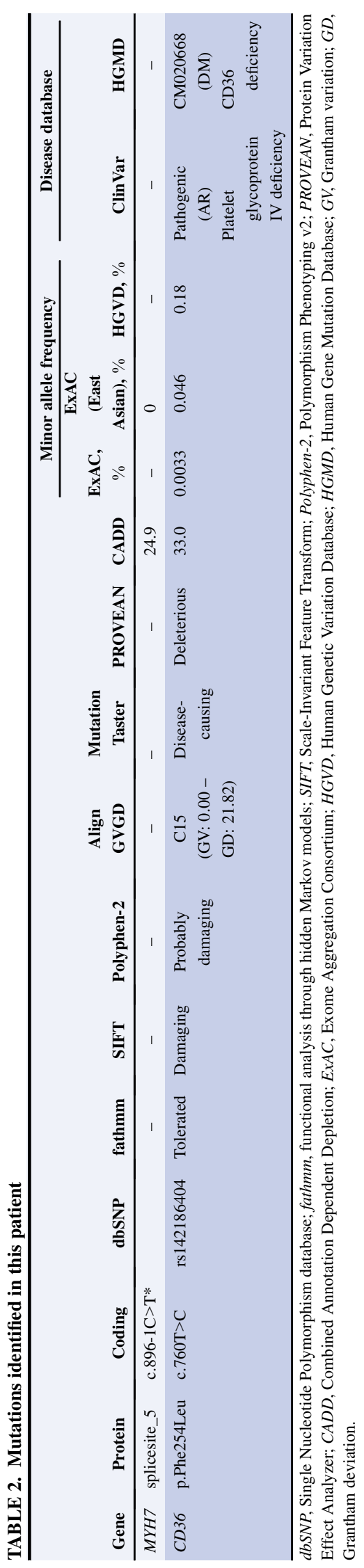

\section{References}

1. Chin TK, Perloff JK, Williams RG, Jue K, Mohrmann R. Isolated noncompaction of left ventricular myocardium. A study of eight cases. Circulation. 1990;82:507-13.

2. Hanawa H, Watanabe K, Nakamura T, Ogawa Y, Toba K, Fuse I, et al. Identification of cryptic splice site, exon skipping, and novel point mutations in type I CD36 deficiency. J Med Genet. 2002;39:286-91.

3. Hoedemaekers YM, Caliskan K, Michels M, Frohn-Mulder I, van der Smagt JJ, Phefferkorn JE, et al. The importance of genetic counseling, DNA diagnostics, and cardiologic family screening in left ventricular noncompaction cardiomyopathy. Circ Cardiovasc Genet. 2010;3:232-9. 